\title{
Komani [Dalmace] (Albanie)
}

Déroulement de la campagne 2011

\section{Etleva Nallbani, Maël Julien et Elvana Metalla}

Institut archéologique d'Albanie, Ministère des Affaires étrangères et européennes, École française de Rome, CEPAM (UMR 6130 UNS-CNRS Nice), INED (Paris), Centre d'histoire et civilisation de Byzance (UMR 8167 - «Orient et Méditerranée » et Paris) (éd.)

\section{(2) OpenEdition Journals}

Édition électronique

URL : http://journals.openedition.org/cefr/695

DOI : 10.4000/cefr.695

ISSN : 2282-5703

\section{Éditeur}

École française de Rome

Référence électronique

Etleva Nallbani, Maël Julien et Elvana Metalla, « Komani [Dalmace] (Albanie) », Chronique des activités archéologiques de l'École française de Rome [En ligne], Balkans, mis en ligne le 19 décembre 2012, consulté le 12 septembre 2019. URL : http://journals.openedition.org/cefr/695; DOI : 10.4000/ cefr.695

Ce document a été généré automatiquement le 12 septembre 2019.

(c) École française de Rome 


\title{
Komani [Dalmace] (Albanie)
}

Déroulement de la campagne 2011

\author{
Etleva Nallbani, Maël Julien et Elvana Metalla
}

Institut archéologique d'Albanie, Ministère des Affaires étrangères et européennes, École française de Rome, CEPAM (UMR 6130 UNS-CNRS Nice), INED (Paris), Centre d'histoire et civilisation de Byzance (UMR 8167 - «Orient et Méditerranée » et Paris) (éd.)

\section{NOTE DE L'ÉDITEUR}

La mission franco-albanaise de Komani est dirigée par Etleva Nallbani (archéologue UMR 8167, « Orient et Méditerranée ", Paris). Ont participé à cette campagne huit spécialistes et trois étudiants (E. Nallbani ; E. Metalla, archéologue et céramologue, à l'Institut archéologique de Tirana ; M. Julien, architecte-archéologue à la DAPCAD ; S. Lela, archéologue à l'AshA; Gj. Gjuraj, archéologue au Directorat régional des Monuments historiques de Durrës ; H. Sokoli, archéologue au Parc archéologique de Shkodra ; E. Bitri, restaurateur à l'ARCS ; D. Margjoni, restaurateur du Parc archéologique de Lezha ; A. Kurrizo, étudiant à l'Université de la Sapienza, Rome ; N. Paireau, étudiant en topographie à l'INSA). Cinq techniciens et une vingtaine d'ouvriers ont secondé la mission et trois animaux ont assuré le transport du matériel.

Le programme quadriennal est soutenu et financé, pour la France, par le Ministère des Affaires étrangères et européennes et par l'École française de Rome, avec une contribution du Centre d'Études Préhistoire, Antiquité, Moyen Âge (UMR 6130, UNS-

CNRS, Nice), de l'Institut national d'études démographiques (Paris) et du Centre d'histoire et civilisation de Byzance (UMR 8167 - « Orient et Méditerranée », Paris).

1 Les opérations de terrain sur le site de Komani (Dalmace) ont été menées au cours de quatre semaines, du 3 août au 2 septembre $2011^{1}$.

2 À l'issue de la campagne de l'an dernier, nous avions prévu de concentrer nos efforts sur la fouille de trois composants du site : l'habitat (zone 1), la nécropole (zones 2 et 3) et le secteur de Saint-Georges (zone 3). Néanmoins, le prévisionnel des opérations, contrarié d'une part par des nécessités de sécurité et d'autre part par l'intervention parallèle à 
caractère de sauvetage sur le site de Lezha ${ }^{2}$, nous a obligé à modifier notre programme en reportant l'exploration de certaines zones (la partie sommitale de la zone 1 de l'habitat et les zones 2 et 3 de la nécropole) pour la campagne suivante. La campagne a poursuivi ainsi ses opérations sur: l'habitat (zone 1), principalement sur deux de ses niveaux, le médian (zone 1A secteurs 1 et 2) et le bas (zone 1B, secteur 3); sur l'église Saint-Georges (fig. 1).

Fig. 1 - Komani. Plan des différentes zones du site sur carte.

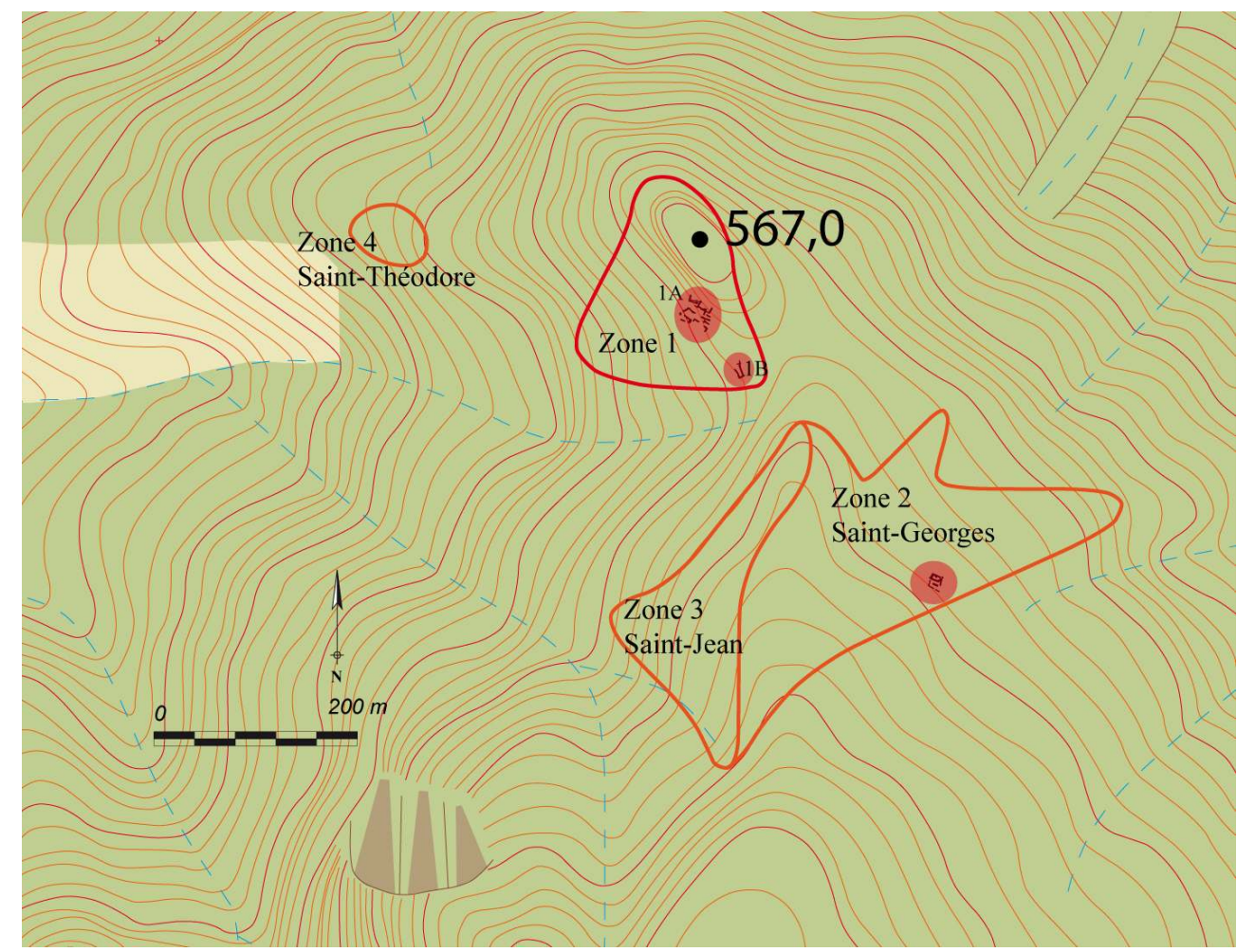

DAO : M. Julien, S. Vatteoni et N. Paireau.

Niveau médian de l'habitat, zone $1 \mathrm{~A}$ - secteurs 1 et 2 (fig. 2) 
Fig. 2 - Komani. Plan de la fouille des secteurs 1 et 2 de la zone $1 \mathrm{~A}$.

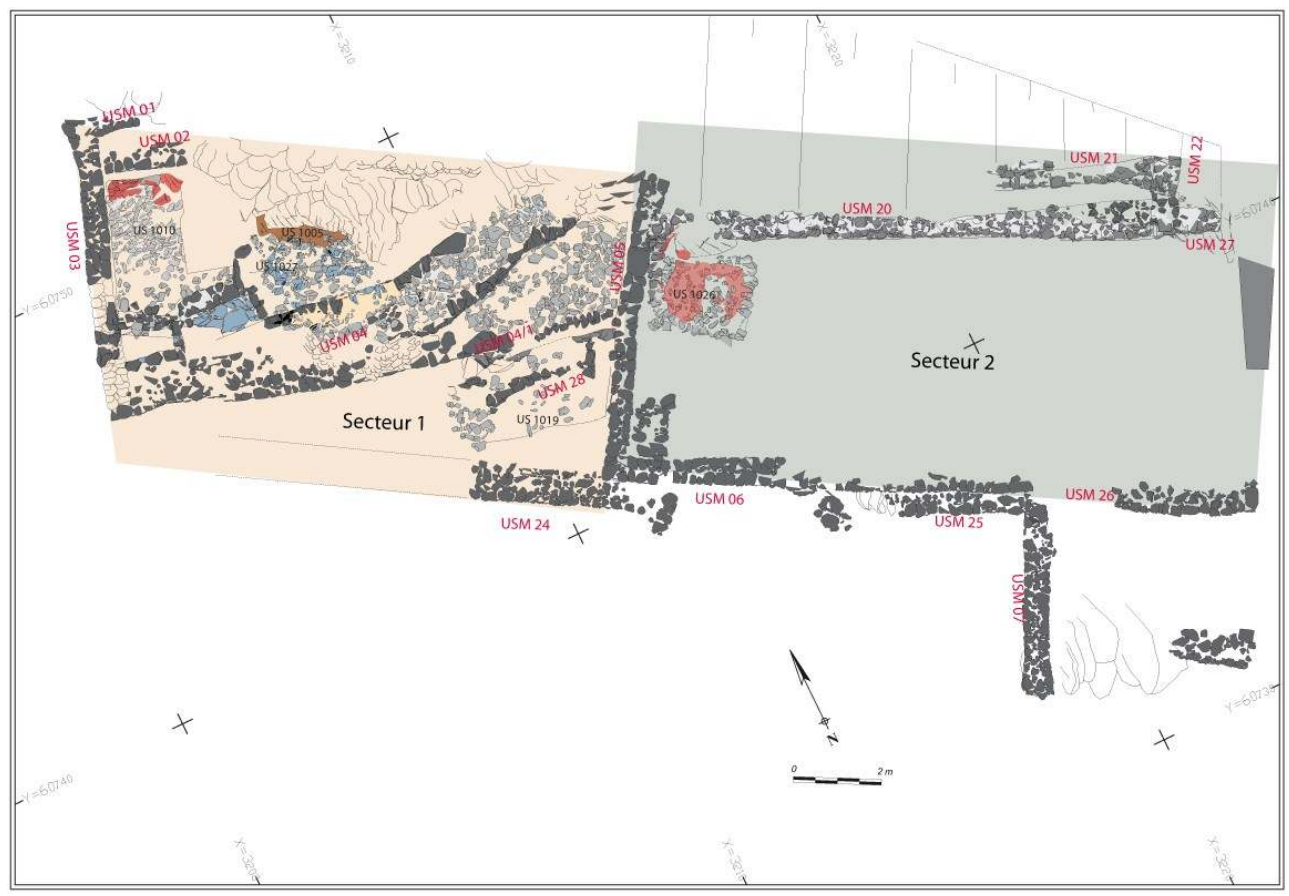

À la fin de la campagne 2010.

DAO : M. Julien, N. Paireau.

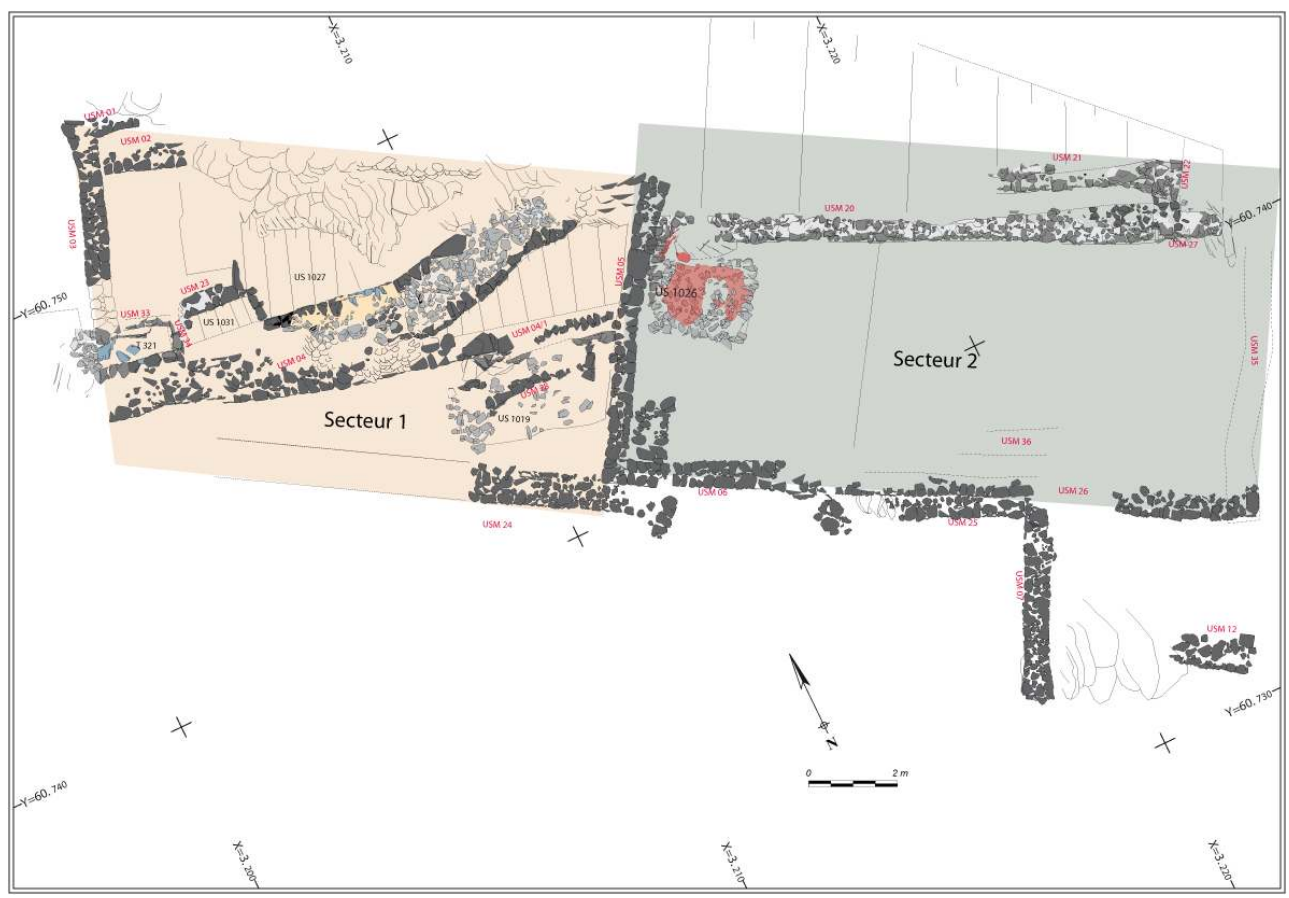

À la fin de la campagne 2011

DAO : M. Julien, N. Paireau.

Dans le secteur 1, les opérations ont eu pour objectifs: la compréhension de l'organisation interne de la pièce encadrée par les USM 02, 03 et 04 ; l'identification et la caractérisation des différentes composantes (USM, tombes et sols) et leur relations chronologiques. Le niveau de colmatage (US 1023) a été complètement dégagé jusqu'aux 
fondations entre la paroi rocheuse au nord et l'USM 04. L'attribution du mobilier résiduel à la période antique et tardo-antique (II siècle avant J.-C. au IV siècle après J.-C.) a été confirmée. On conclut ainsi que la première structuration du secteur $1 \mathrm{a}$ eu lieu à la fin de l'Antiquité tardive, faisant usage d'un mobilier résiduel issu d'une occupation plus ancienne, dont les témoins de constructions demeurent absents à l'heure actuelle.

Il était important de comprendre l'usage des deux structures rectangulaires, installées contre l'USM 04, et leurs relations fonctionnelles et chronologiques avec l'espace du secteur 1. La fonction domestique de cette dernière a déjà été reconnue lors des précédentes campagnes. Les deux structures sont disposées selon le même axe, dont une des deux (T.330, US 1030 et 1031) s'ouvre avec certitude dans le niveau de colmatage (US 1023).

5 La première, déjà identifiée en tant que sépulture (T. 321), a été partiellement fouillée en 2010. Elle représente une structure maçonnée (pierres liées au mortier de chaux) sur les trois côtés nord (USM 33), sud (USM 04) et est (USM 33). Sa limite ouest passe au dessous de l'USM 03 pour s'appuyer sur la roche naturelle. La sépulture mesure 2,38 $\mathrm{m}$ de long sur $0,86 \mathrm{~m}$ de large et $0,80 \mathrm{~m}$ de profondeur. Nous avions, en 2010, seulement dégagé les dépôts osseux secondaires situés dans l'angle est de la structure. Au moins sept individus, dont l'étude anthropologique est en cours, ont été identifiés (fig. 3).

Fig. 3 - Komani. La sépulture 321.

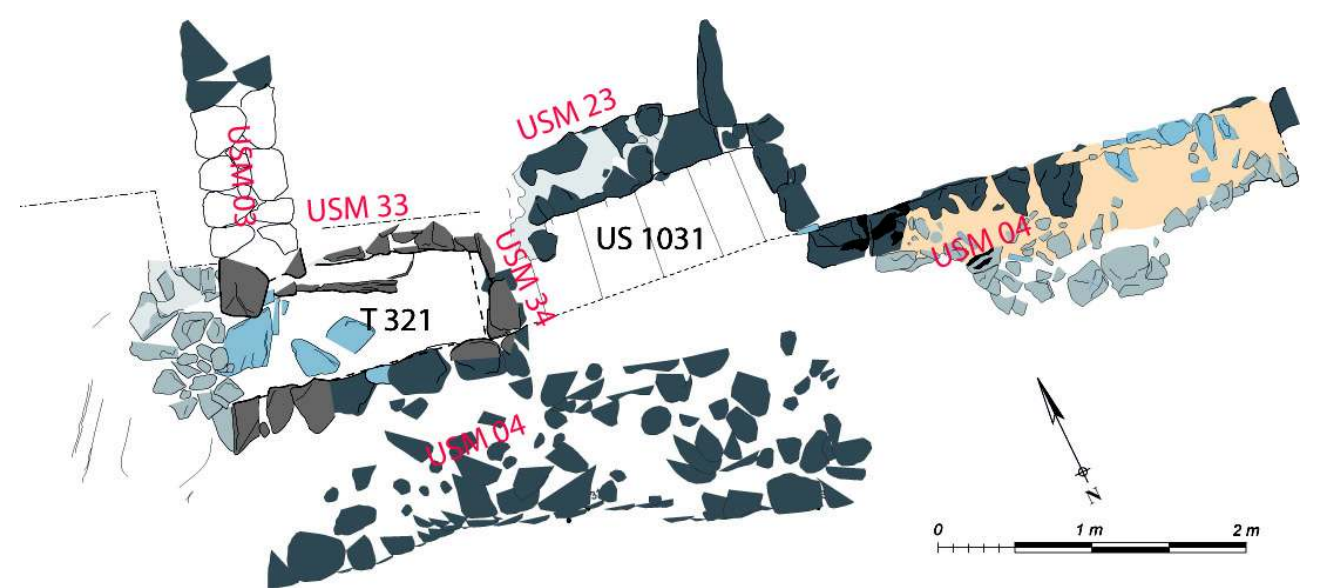

Plan. 


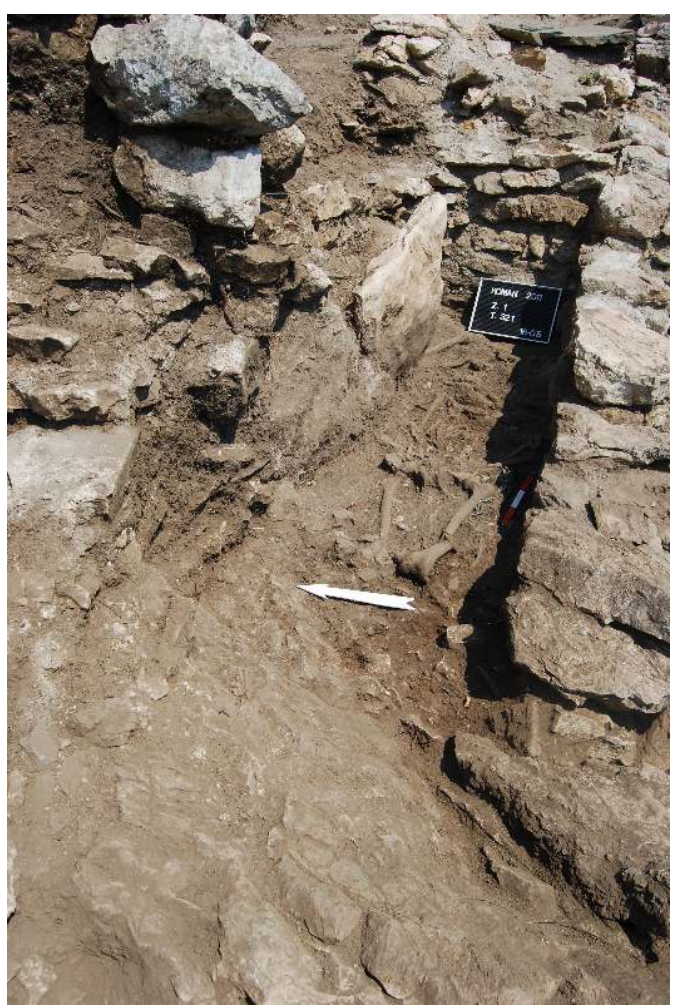

Inhumation partielle.

Cliché E. Nallbani.

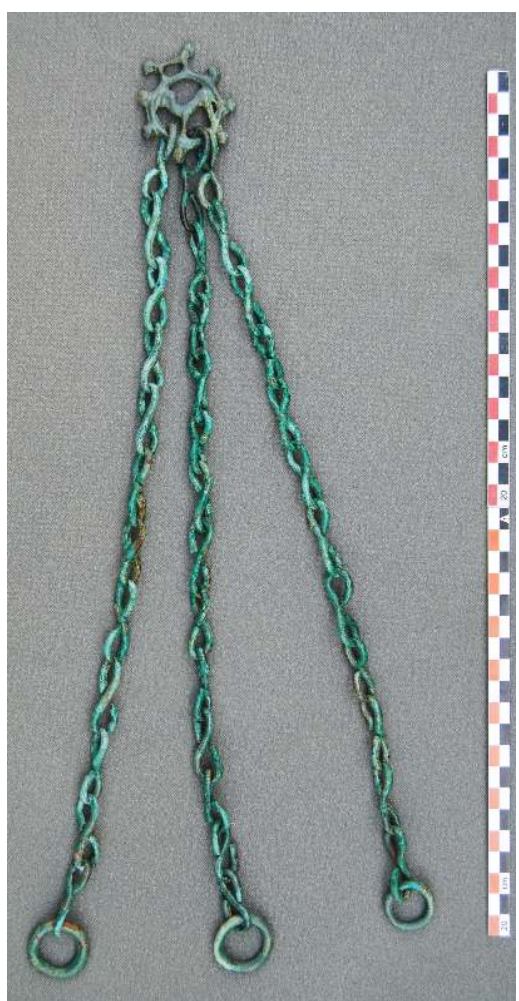

Objet du mobilier, châtelaine.

Cliché E. Nallbani.

Lors de notre dernière campagne, la sépulture nous a livré, outre les restes des dépôts osseux secondaires, une inhumation primaire en décubitus dorsal, tête à l'est. Ce 
squelette a subi de sérieux bouleversements : les membres inférieurs étaient conservés jusqu'aux genoux et la partie supérieure du crâne faisait défaut. Cela est probablement dû au réaménagement du secteur 1 , situé vers les $\mathrm{X}^{\mathrm{e}}-\mathrm{XI}^{\mathrm{e}}$ siècles, rajoutant notamment la construction de l'USM 03, qui empiète sur la partie ouest du fond de la tombe. Deux des dalles de couverture conservées s'appuyaient sur chant sur l'USM 33.

7 L'individu de sexe féminin était accompagné d'un mobilier vestimentaire particulièrement riche et relativement bien conservé : deux paires d'anneaux temporaux en anneaux circulaires de bronze avec pendentif en fil torsadé; une paire de boucles d'oreilles en argent dont chacune est composée d'un anneau circulaire muni d'un œillet de fermeture et d'un pendentif formé d'une calotte semi-sphérique décorée de quatre pétales en filigrane; un torque formé d'une tige de bronze torsadée avec un œillet de fermeture et, en pendentif, un grelot de même métal, pourvu d'une ouverture en croix ; cent-quarante-six perles en pâte de verre de différents types et couleurs (millefiori, aux ocelles, tubulaires, sphériques et segmentées) et en améthyste; une bague en bronze montée en bâte (chaton en argent et pâte de verre) ; une châtelaine à l'anneau ajouré avec motif de cheval d'où pendent trois chaînes aux maillons en forme de 8 qui se terminent en anneaux circulaires (le tout en bronze) et un couteau en fer avec des restes de son étui en bois. Cette parure trouve une répartition très étendue, du nord Caucasien (la région de Volga-Kama) jusqu'en Europe centrale, notamment dans la plaine Pannonienne. Certains éléments comme la châtelaine et le torque sont caractéristiques du costume asiatique dit de Saltovo et des Avars tardifs. L'ensemble du mobilier de cette inhumation primaire pourrait être daté du dernier tiers du VII ${ }^{\mathrm{e}}$ siècle et tout au long du VIII ${ }^{\mathrm{e}}$ siècle ${ }^{3}$.

On constate la pratique de l'incinération partielle sur le défunt, phénomène observé pour la première fois parmi les rites funéraires régionaux des $\mathrm{VII}^{\mathrm{e}}-\mathrm{VIII}{ }^{\mathrm{e}}$ siècles. La quantité considérable de charbon de bois et de graines carbonisées étalés au dessous de l'inhumation suggère le dépôt du défunt sur un lit de braises incandescentes; des fragments d'ossements et des perles carbonisés à des températures relativement basses en témoignent également. Par endroit, la terre de remplissage est très noire; certains éléments du mobilier ont pris le même aspect: le chaton de la bague, le torque et les boucles d'oreilles, dont les feuilles fines des calottes en argent ont subi une dissolution partielle. En l'état actuel de nos études, il n'est pourtant pas possible de déterminer si ce processus de combustion a été réalisé in situ. La roche naturelle déterminait le fond de la sépulture qui suit la pente du terrain sans traces d'aménagement particulier.

Il semble indéniable que l'installation de la sépulture (T. 321) contre l'USM 04 a eu lieu avant que les secteurs 1 et 2 ne soient l'objet du second aménagement (phase V), attribué aux $\mathrm{X}^{\mathrm{e}}-\mathrm{XI}^{\mathrm{e}}$ siècles ${ }^{4}$. Ceci est attesté par la construction de l'USM 03 qui empiète sur la partie occidentale du fond de la sépulture et sur la couche d'effondrement de toiture, cette dernière datée par le mobilier céramique des $\mathrm{V}^{\mathrm{e}}$-VII ${ }^{\mathrm{e}}$ siècles, tout comme l'US 1027 dégagée en 2010 sur les niveaux de sol 1013 et 1014. Cet effondrement a par ailleurs été observé à l'ouest, au delà de l'USM 03 (fig. 4), indiquant ainsi l'extension occidentale du secteur 1 lors du premier aménagement et que sa disposition n'a pas été prise en compte lors de son réaménagement des $\mathrm{X}^{\mathrm{e}}-\mathrm{XI}$ siècles. 
Fig. 4 - Komani. Secteur 1 de la zone A, partie nord-ouest vue du sud.

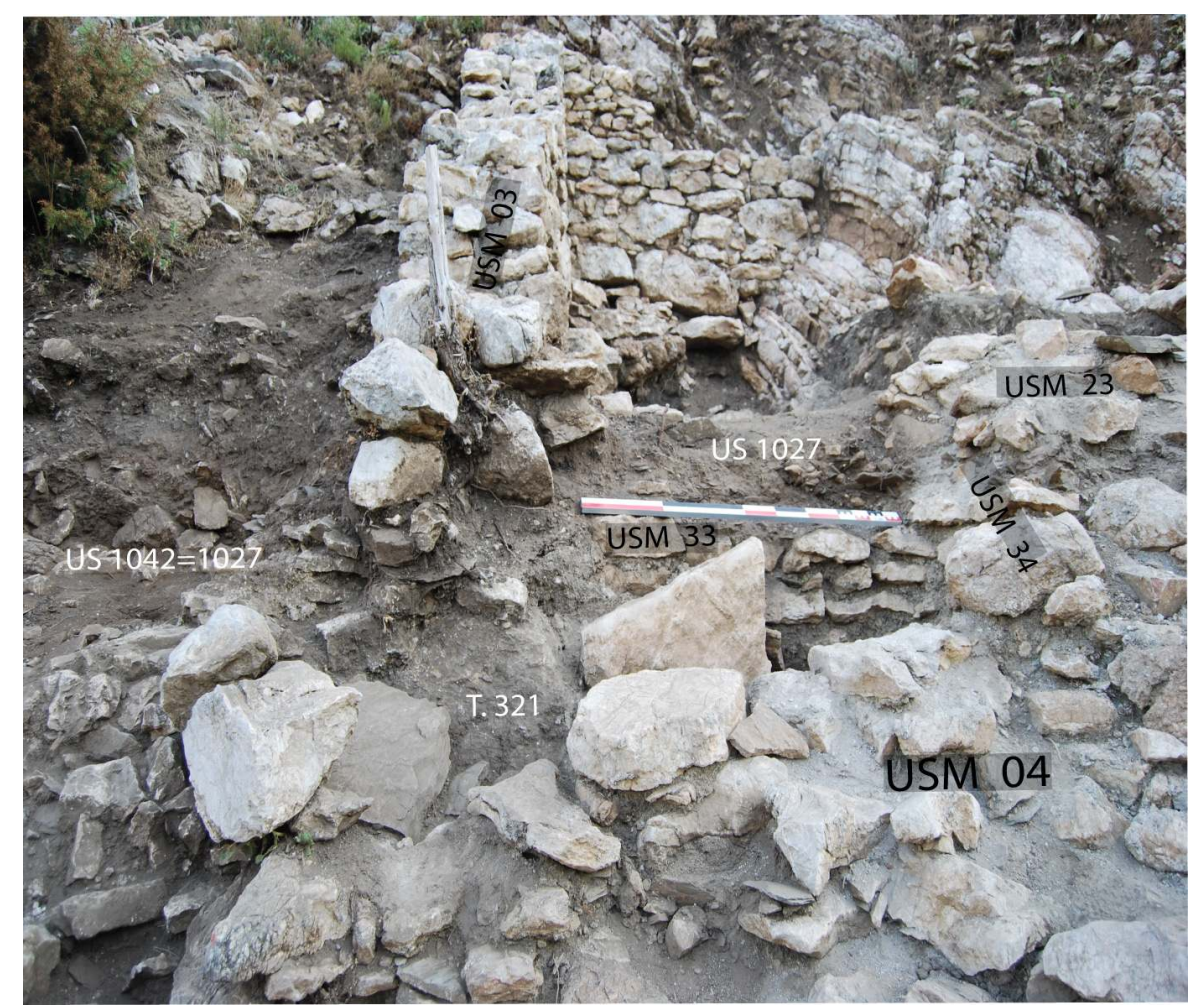

Cliché E. Nallbani.

Cependant, le rapport stratigraphique entre le premier aménagement du secteur (situé à la fin de l'Antiquité) et l'installation de la sépulture reste à clarifier. La possibilité d'une installation de la sépulture T. 321 avant le premier aménagement du secteur 1, c'est-àdire avant la fin de l'Antiquité tardive (US 1013, 1014 et 1027), est à écarter au regard de l'attribution chronologique du mobilier de l'inhumation primaire (dernier tiers du VII VIII ${ }^{\mathrm{e}}$ siècle). Deux hypothèses sont possibles. La première pourrait lui attribuer un usage contemporain à celui de l'occupation domestique. Dans ce cas, nous serions en présence d'une structure ouverte de type appentis, attribuant à l'USM 04 un rôle de soutènement et à l'ensemble de l'espace compris entre les USM 04, 02 et 03 celui d'une terrasse. La seconde hypothèse attribue l'installation de la sépulture après la première destruction du secteur (phase IV), ce qui signifierait l'abandon total des structures jusqu'à la seconde réorganisation $\mathrm{du}$ secteur des $\mathrm{X}^{\mathrm{e}}-\mathrm{XI}^{\mathrm{e}}$ siècles, ou bien l'abandon de leur fonction domestique au profit d'autres fonctions, y compris la funéraire. En l'état actuel de la recherche, et compte tenu de l'état de conservation des vestiges (puisque l'extrémité sudouest du secteur $1, \mathrm{y}$ compris l'USM 04, ont été sérieusement endommagés par le glissement prononcé du terrain), il est impossible de commenter davantage ces deux hypothèses. Les implications de ces hypothèses pour ce secteur de l'habitat médian seraient très importantes dans la compréhension de la transformation des fonctions de l'ancien habitat tardo-antique à l'époque médiévale.

11 La seconde structure maçonnée (US 1031) selon le même axe que la tombe 321, le long du mur USM 04, avait également été envisagée en 2010 comme une structure funéraire (fig. 5). 
Fig. 5 - Komani. Secteur 1 de la zone 1A, partie nord-ouest.

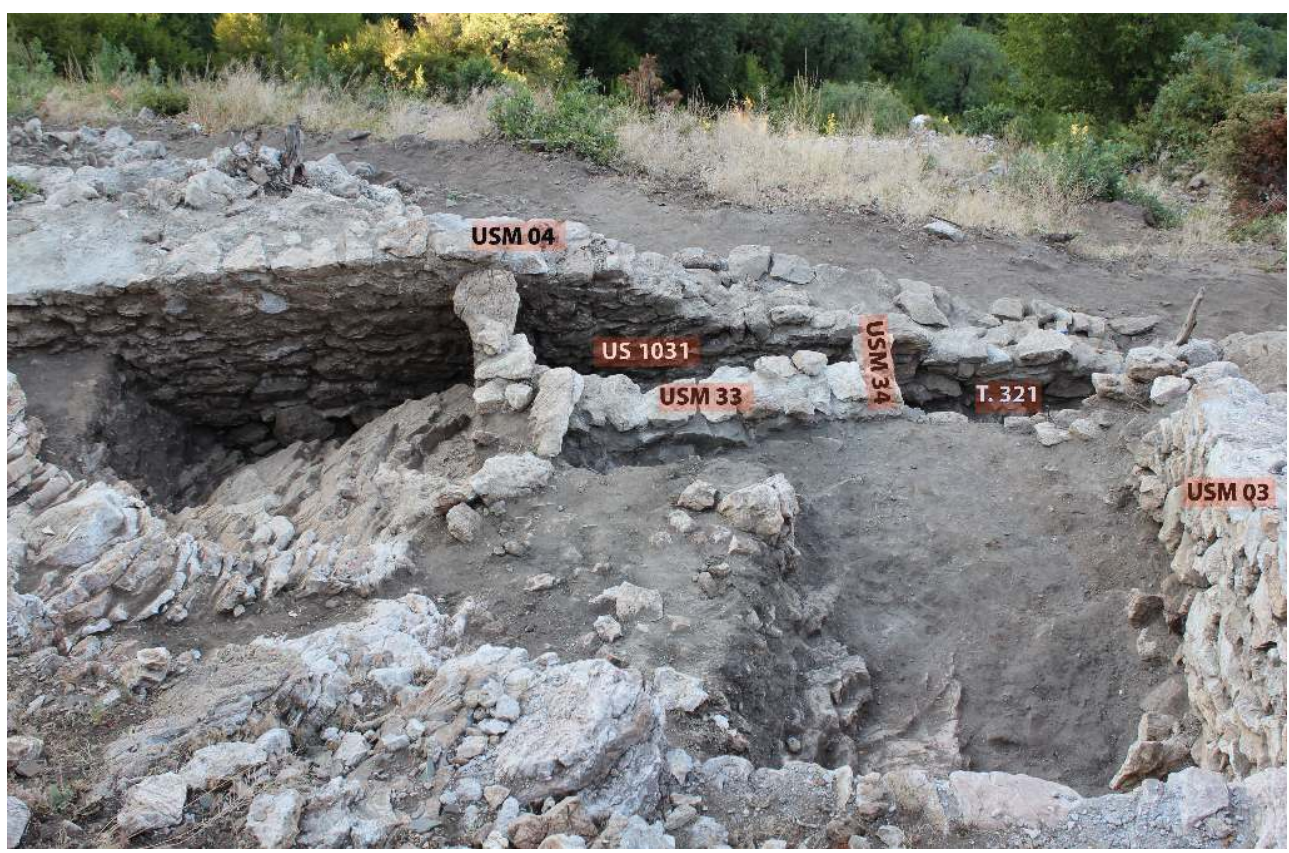

Cliché E. Nallbani.

En effet, l'aménagement consistait en un espace grossièrement rectangulaire de 1,42 $\mathrm{m}$ de long sur 0,63 $\mathrm{m}$ de large, délimité par l'USM 23, épais de 0,50 $\mathrm{m}$. Ce mur, positionné parallèlement à l'USM 04, est constitué seulement de trois assises de pierres calcaires liées au mortier de chaux. Sur le côté est, la structure s'appuie sur la roche naturelle et rejoint l'USM 04 par l'intermédiaire de trois pierres faiblement liées au mortier, posées directement sur le niveau de remplissage, sur la couche de dalles en ardoise (l'effondrement de la toiture). L'extrême ouest de l'USM 23 crée un angle également maçonné (USM 34) qui empiète sur la limite est de la tombe 321. Sa postériorité à la construction de la tombe 321 ne fait pas de doute.

13 Après avoir enlevé les dalles en ardoise posées à plat à l'intérieur de l'espace rectangulaire de cette structure, une couche de petites pierres mélangées à de petits blocs de mortier reposait sur un remblai de terre marron foncé. Cette dernière a livré une quantité considérable d'ossements d'animaux et de rares tessons de céramiques, datables des $\mathrm{V}^{\mathrm{e}}$-VIII ${ }^{\mathrm{e}}$ siècles. La roche naturelle a été atteinte, elle rejoint quasiment les fondations de l'USM 04. Après l'achèvement de la fouille de la structure 1031, il semble que celle-ci ne puisse plus être envisagée en tant que sépulture, d'autant que son remplissage paraît très similaire à l'US 1023 , ce qui pourra situer sa construction après la première destruction du secteur, à la fin de l'Antiquité tardive. Quelle est alors la destination de cet aménagement, dont l'élévation ne dépasse pas celle de l'USM 04 ? Aurait-il assuré un rôle de plate-forme devant un accès, ou celui d'un palier d'escalier conduisant vers un étage supérieur?

14 Toujours dans le secteur 1, cette fois-ci dans sa moitié est (fig. 6), les opérations ont visé à mieux comprendre la morphologie des structures, et à cerner les éventuelles relations chronologiques entre elles. 
Fig. 6 - Komani. Secteur 1 de la zone 1 A à l'issue de la campagne 201 1, partie sud-est vue du nordest.

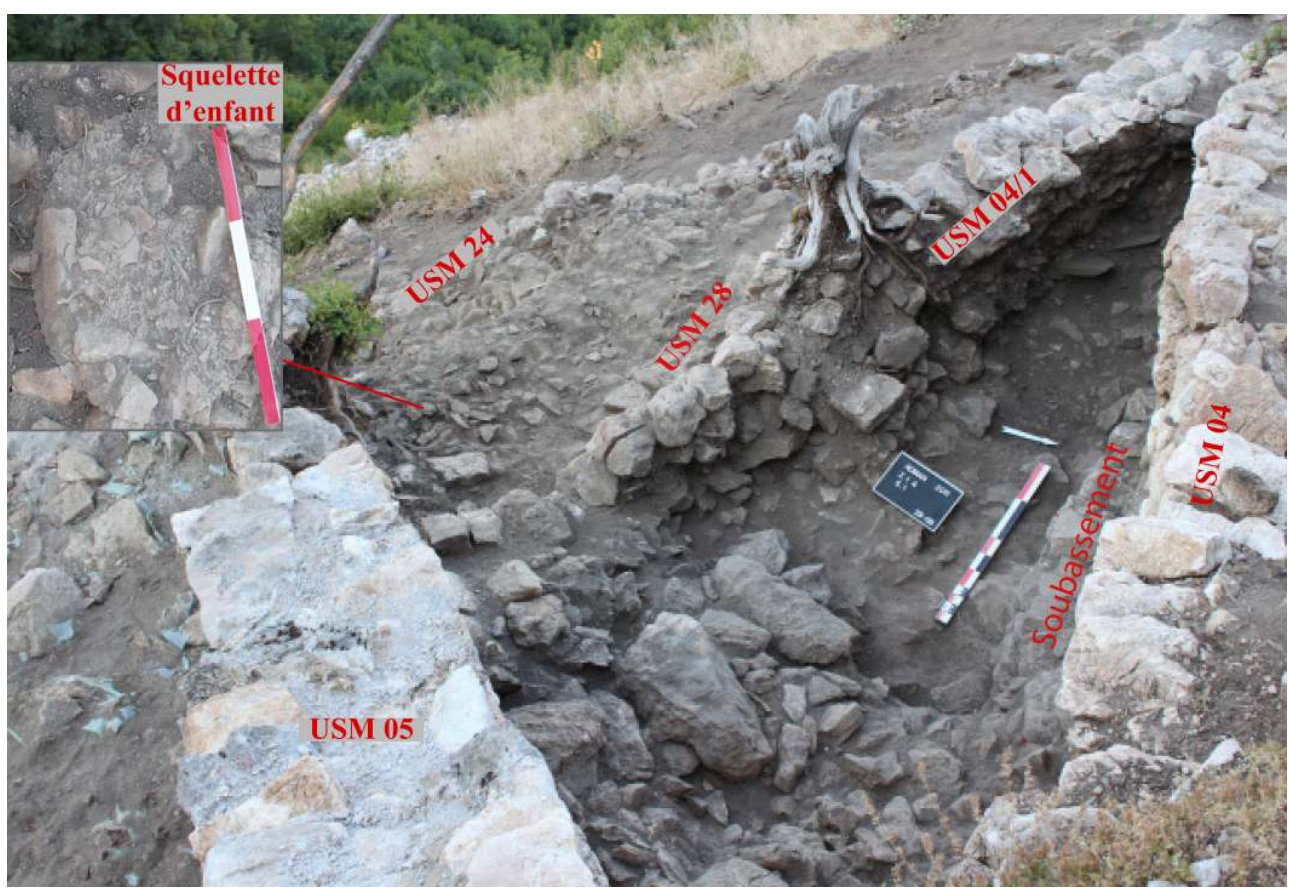

Cliché E. Nallbani.

Il s'agit notamment de comprendre l'organisation de deux terrasses, par la fouille de la terrasse encadrée par les USM 04, 04/1 et 05 et de celle comprise entre les USM 24, 05 et $04 / 1$.

16 À l'issue de la campagne 2010, nous avions identifié, dans cette dernière terrasse, un niveau de seuil (US 1019), allant de pair avec un alignement de quatre pierres maçonnées (USM 28), tous deux étant attribués à la première structuration du secteur 1 (entre le $\mathrm{V}^{\mathrm{e}}$ et le $\mathrm{VII}^{\mathrm{e}}$ siècle), quand l'accès était assuré par l'ouverture dans l'USM 05. Auprès d'un premier squelette d'enfant âgé de 0 à 6 mois, découvert en 2010 sous le niveau de seuil, le nettoyage de cette année a mis au jour les ossements d'un deuxième enfant, dont l'âge est à définir. Cette terrasse fera objet d'une fouille complète lors de la prochaine campagne.

L'achèvement de la fouille de la première terrasse, encadrée donc par les USM 04, 04/1 et 05 , a révélé un mur 04/1 premièrement intégré dans le mur USM 04 pour se séparer ensuite et s'appuyer sur l'USM 05. Il est également constitué de pierres liées au mortier de chaux, à deux faces régulières, jusqu'à $2 \mathrm{~m}$ de la jonction avec L'USM 04 . Son élévation prend ensuite un aspect irrégulier avec la raréfaction du liant et rejoint l'USM 05. La fouille de cette année a atteint le niveau de la fondation des deux USM, 04 et 04/1. Un soubassement arqué de deux assises en pierres se trouve au dessous de l'USM 04, sur une longueur de $2 \mathrm{~m}$, consolidant ainsi les fondations de l'USM 04. L'angle formé par ces deux USM a révélé une terre noire et compacte, contenant une quantité considérable d'ossements d'animaux et de fragments de céramique culinaire résiduelle qui pourraient être datés entre le $\mathrm{VII}^{\mathrm{e}}$ et le $\mathrm{X}^{\mathrm{e}}$ siècle. Le reste du remblai de la terrasse presque triangulaire comportait un mélange de pierres de toutes tailles, prises dans une terre meuble, accompagné d'une faible quantité d'ossements d'animaux ainsi que de fragments de céramique peu nombreux. Toutefois, le mur 04/1 peut vraisemblablement être associé aux derniers états d'aménagement du secteur 1 . Cette maçonnerie semble constituer la 
façade sud d'un ensemble rectangulaire défini par la paroi rocheuse et le mur 01 au nord, 03 à l'ouest et 05 à l'est. En l'état actuel de la recherche, il ne nous est pas possible de définir la date précise de la construction de cette terrasse. Nous la voyons plutôt contemporaine au second réaménagement du secteur 1 , compte tenue de la datation du matériel céramique résiduel ainsi que des indications sur l'existence de murs pris dans les fondations des USM 04 et 04/1. Mais pour clarifier cette situation, il faudra prévoir des démontages partiels des deux murs.

Dans le secteur 2 de la même zone 1A (l'habitat médian), la fouille s'est investie dans la compréhension de l'organisation de cet espace qui s'est révélé assez vaste (fig. 7).

Fig. 7 - Komani. Secteur 2 de la zone 1A, vue prise de l'est à la fin de la campagne 2011.

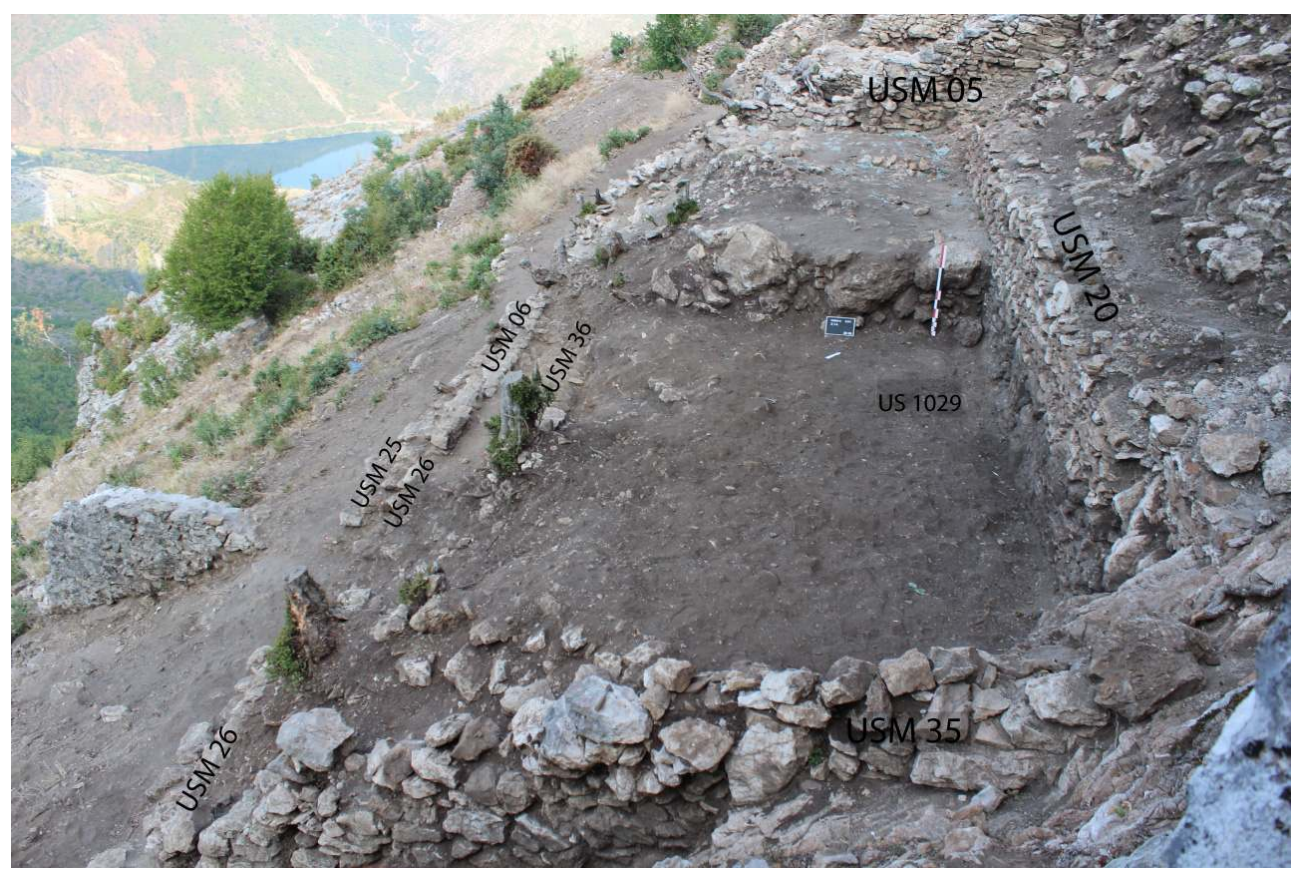

Cliché E. Nallbani.

19 Ce secteur rectangulaire, d'environ $105 \mathrm{~m}^{2}$, est délimité par plusieurs murs sur les côtés nord, ouest et sud (les USM 20, 25, 26, 06) et par la roche naturelle. Le niveau d'occupation identifié en 2010 fut attribué au titre d'hypothèse aux Xe-XIe siècles, avec un four quasicirculaire installé à l'angle nord-ouest du secteur 2. La majorité de la partie est de l'espace (d'environ $75 \mathrm{~m}^{2}$ ) a été fouillée en 2011, jusqu'à $0,50 \mathrm{~m}$ de profondeur, atteignant la fondation de l'USM 20. L'ensemble présentait un remplissage constitué de pierres (US 1029) ou de gros blocs de roche. Peu de mobilier céramique a été fourni, de très rares fragments d'ossements d'animaux et seulement une anse de récipient en fer. Il s'agit apparemment de tout un niveau préparatoire d'occupation, qui correspond à celui de l'installation du four.

20 Il est surprenant l'absence de cloisonnement en directions attendus plutôt nord-ouest/ sud-est sur l'ensemble de l'espace, à l'exception de l'USM 35 à l'extrémité est, nouvellement découvert. Ce mur, large de $0,60 \mathrm{~m}$, bâti de pierres non traitées liées au mortier de chaux et dirigé nord-est/sud-ouest, s'appuie tout d'abord sur la roche naturelle pour descendre le long de la pente où il rejoint l'USM 26 dans l'angle sud-sudouest du secteur. On aurait ainsi une sorte d'îlot rectangulaire, qui serait encadré par les 
USM 05, 20, 35 et 26, dont nous n'avons partiellement fouillé que son niveau d'occupation le plus élevé, puisque comme l'ensemble de l'habitat, les structures de ce secteur sont également disposées en espalier. Le nettoyage des USM 26 et 06 a fait apparaître un nouveau mur, l'USM 36, épais de 0,60 m, constitué de pierres liées au mortier et orienté est-ouest, à seulement $0,60 \mathrm{~m}$ au nord de l'USM 26. Ce mur n'a été suivi que sur 2,20 $\mathrm{m}$ de longueur.

Ce secteur oriental de la partie médiane de l'habitat nécessiterait davantage des fouilles intensives pour une meilleure compréhension et interprétation de son organisation. Il comprend probablement plusieurs espaces non encore identifiées, dont les hauteurs conservées ne dépassent pas pour l'instant les niveaux fouillés.

\section{Niveau bas de l'habitat, zone 1B - secteur 3 (fig. 8)}

Fig. 8 - Komani. Vue de la zone 1B.

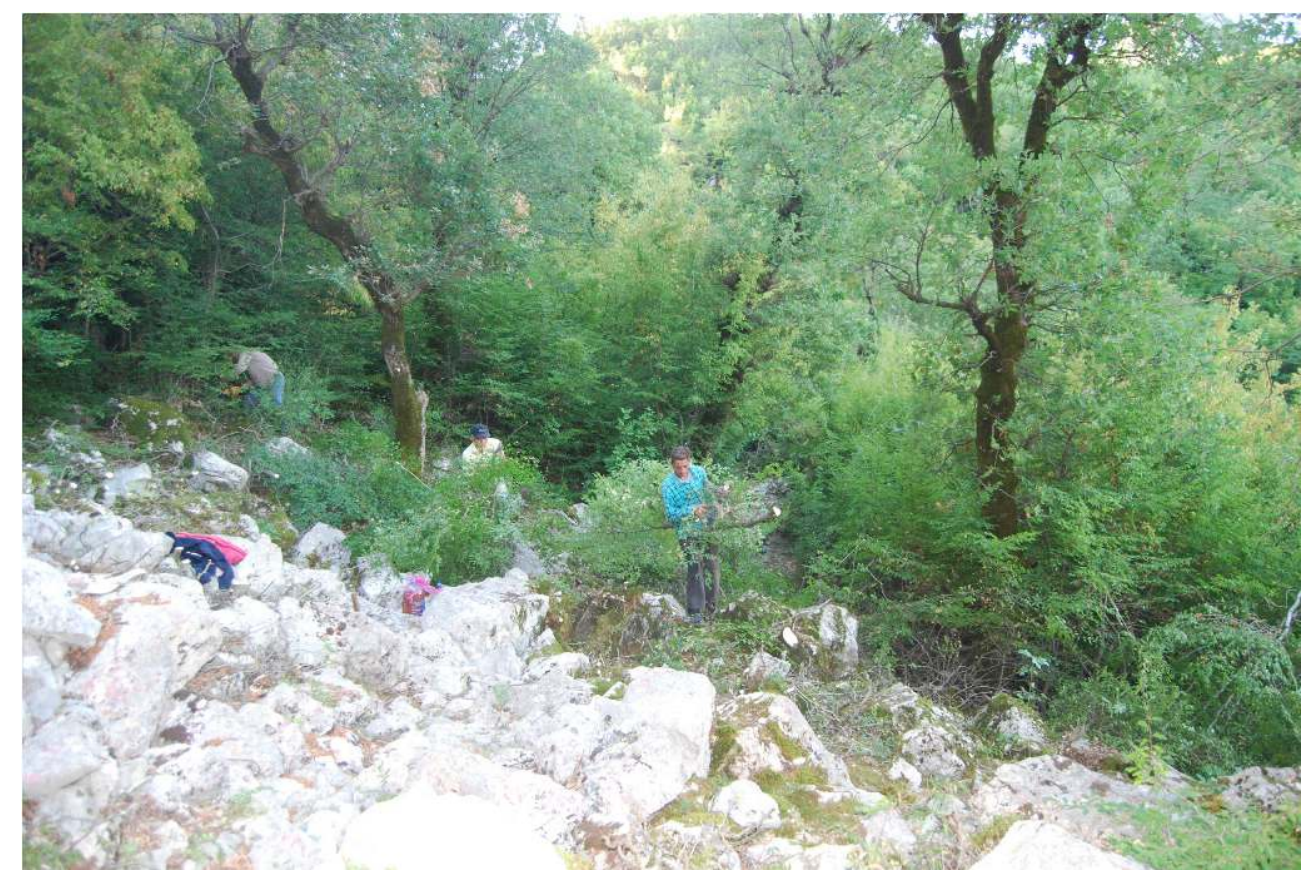

AVANT LA CAMPAgne 2011.

CLICHÉ E. NALLBANI. 


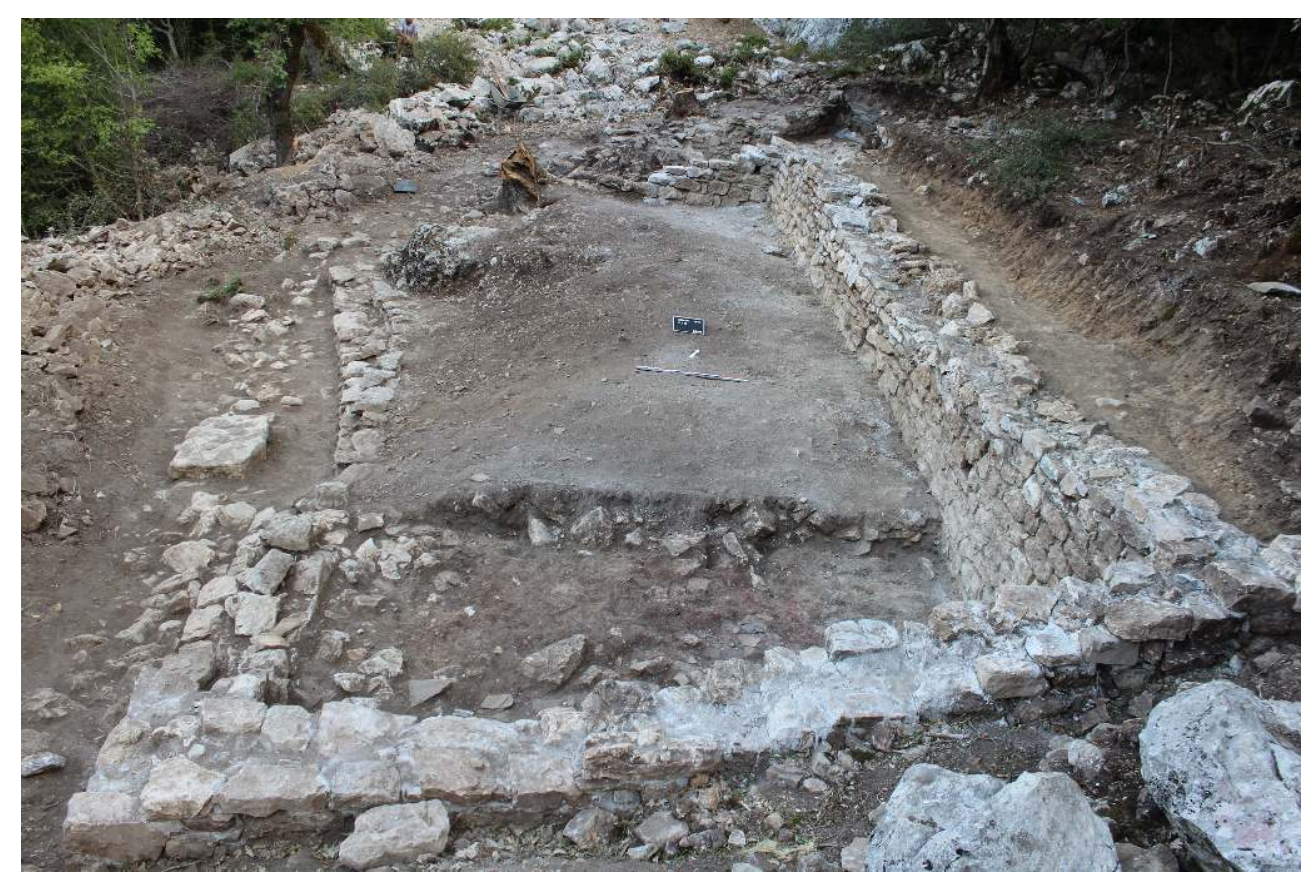

APRÈS LA FOUILLE.

CLICHÉ E. NALLBANI.

Afin de saisir également l'organisation hiérarchique de l'espace occupé du site, par l'intégration des investissements publics, en l'occurrence des remparts, nous avions envisagé le secteur $1 \mathrm{~B}$ en tant qu'entrée de la zone 1, c'est-à-dire l'habitat regroupé du flanc sud-ouest de la colline. Cette nouvelle zone, située en contrebas au sud-est de la colline, a fait l'objet d'importants travaux de nettoyage (qui ont débuté en 2010) : la déforestation et le déblaiement de gros blocs de roche naturelle sur une surface d'environ $230 \mathrm{~m}^{2}$.

23 Le secteur s'est révélé être organisé en trois terrasses successives, en direction nordouest/sud-est, dont seule la terrasse médiane a été dégagée par la fouille en 2011. Celle-ci a livré plusieurs structures en pierres liées au mortier de chaux, dont l'élévation la mieux conservée (l'USM 41) atteint 1,42 m au-dessus du niveau actuel de fouille.

L'ensemble des élévations, dont certaines dotées d'un appareillage soigné, dessine un grande espace d'environ $70 \mathrm{~m}^{2}$ compris entre cinq murs (les USM 38 à 42, fig. 9). 
Fig. 9 - Komani. Plan la fouille de la zone 1B, secteur 3.

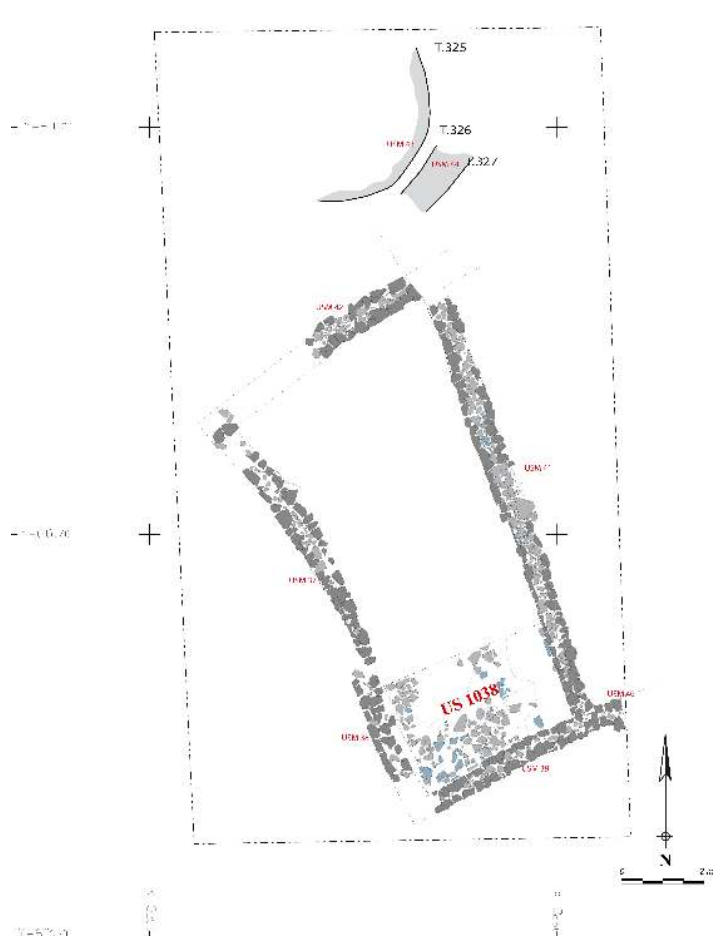

DAO : M. Julien.

L'USM 37 comporte deux rangs de pierres en espalier, dont le premier est constitué de deux assises de pierres non régulières et sans liant, disposées en forme d'arc. La deuxième assise comporte seulement une rangée de pierres, empiétant sur la première et suivant le même arc.

L'USM 41 est long de $11 \mathrm{~m}$, orienté nord-ouest/sud-est. Les deux autres (USM 39 et 42) sont positionnés aux limites est et ouest de l'ensemble. Ils sont tous trois épais de 0,60 m, bâtis pour l'essentiel avec des pierres de moyennes dimensions, taillées et disposées en assises régulières. Du mortier de chaux a ponctuellement été utilisé pour lier les pierres de parement au blocage central. Quelques traces d'enduit ont été très ponctuellement identifiées. Le tout donne un aspect soigné à la surface maçonnée qui comporte également six trous de boulin (quatre dans l'USM 41, un dans l'USM 39, un dans l'USM 42), distribués à des distances irrégulières les uns des autres. L'USM 38 comporte seulement trois assises qui sont connues sur une longueur de 2,50 m (fig. 10). 
Fig. 10 - Komani. Élévations des murs de la zone 1B, secteur 3 (USM 41, 42 et 39).

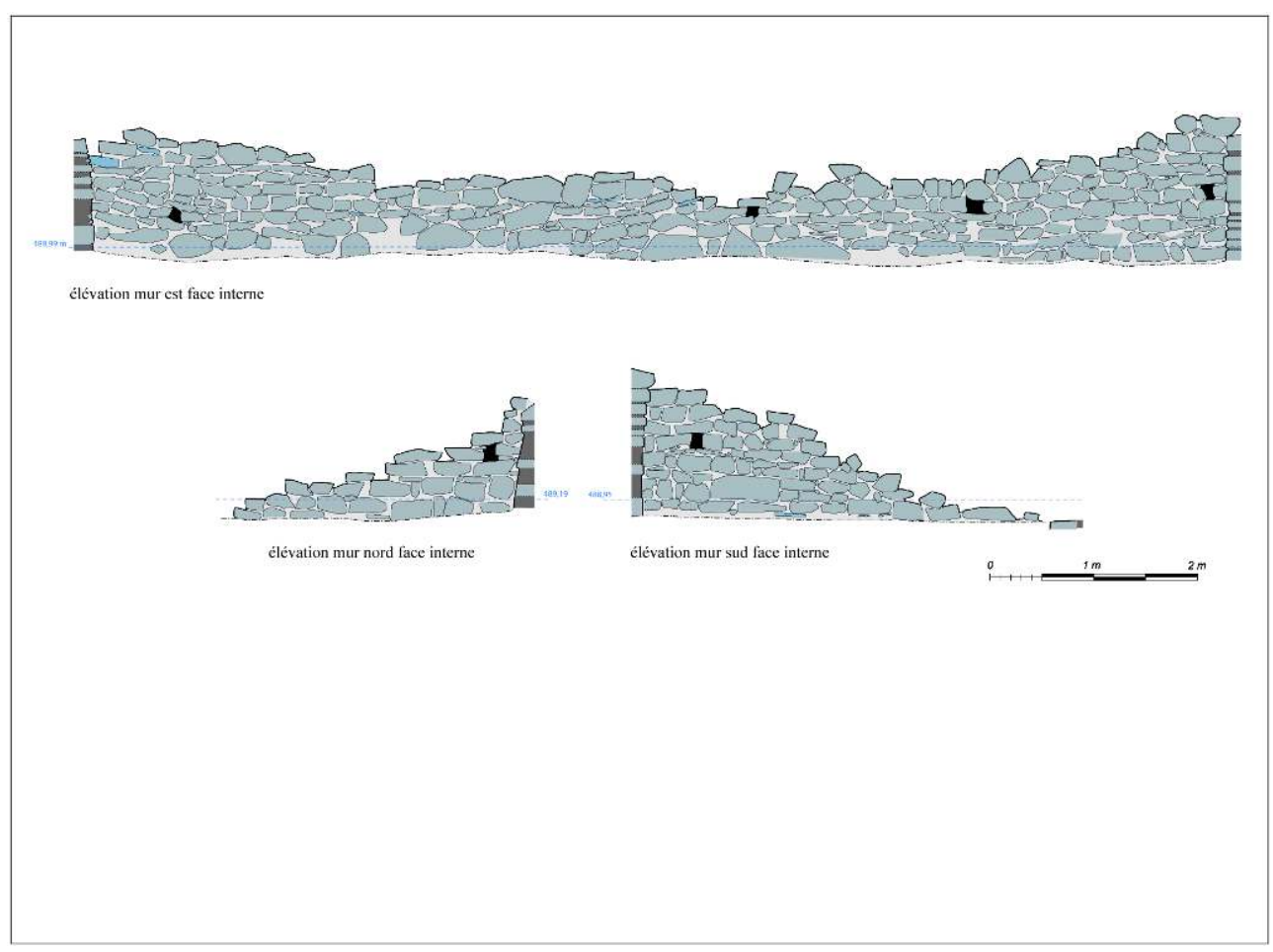

DAO : M. JULIEN.

L'USM 39 semble se poursuivre vers l'est (USM 40) au-delà de l'USM 41. L'extrémité nordouest de l'espace a été particulièrement endommagée par la végétation forestière, dont le mur 42 et sa face externe nord-ouest sont les plus touchés.

Une structure maçonnée est également repérée dans l'angle nord-est.

Les niveaux identifiés au sein de l'ensemble sont constitués en premier lieu d'une épaisse couche de destruction, comportant de gros blocs de roche naturelle, des fragments de murs démolis et de la terre (US 1034). La deuxième couche (US 1036) est plus chargée en ardoises et en petites pierres avec de fins nodules de mortier à proximité des angles. Ces derniers proviennent vraisemblablement d'un enduit de surface.

Nous avons effectué un sondage dans la partie sud-est (d'une surface de 2,5 x $5 \mathrm{~m}$ ) pour comprendre l'usage et les séquences chronologiques de cet ensemble quasi-rectangulaire. Un niveau d'occupation (US 1038) a ainsi été identifié. Il est composé de divers matériaux : roche naturelle polie dans la partie sud-est, pierres et dalles dans la partie sud-ouest, terre battue au centre, quelques fragments d'ardoises. Des traces de chauffe à l'origine indéterminée ont ponctuellement été observées.

31 Les opérations de fouille ont également porté sur la partie nord du secteur, en dehors de l'ensemble de ces structures. L'effondrement de la cavité rocheuse naturelle, matérialisée par la présence massive de très gros blocs, a causé la destruction d'un bâtiment dont certaines élévations ont pu être étudiées. Un tronçon maçonné (USM 43) s'organise selon un arc d'un diamètre interne de 4,30 m pour une épaisseur moyenne de 0,60 m (fig. 11). 
Fig. 10 - Komani.

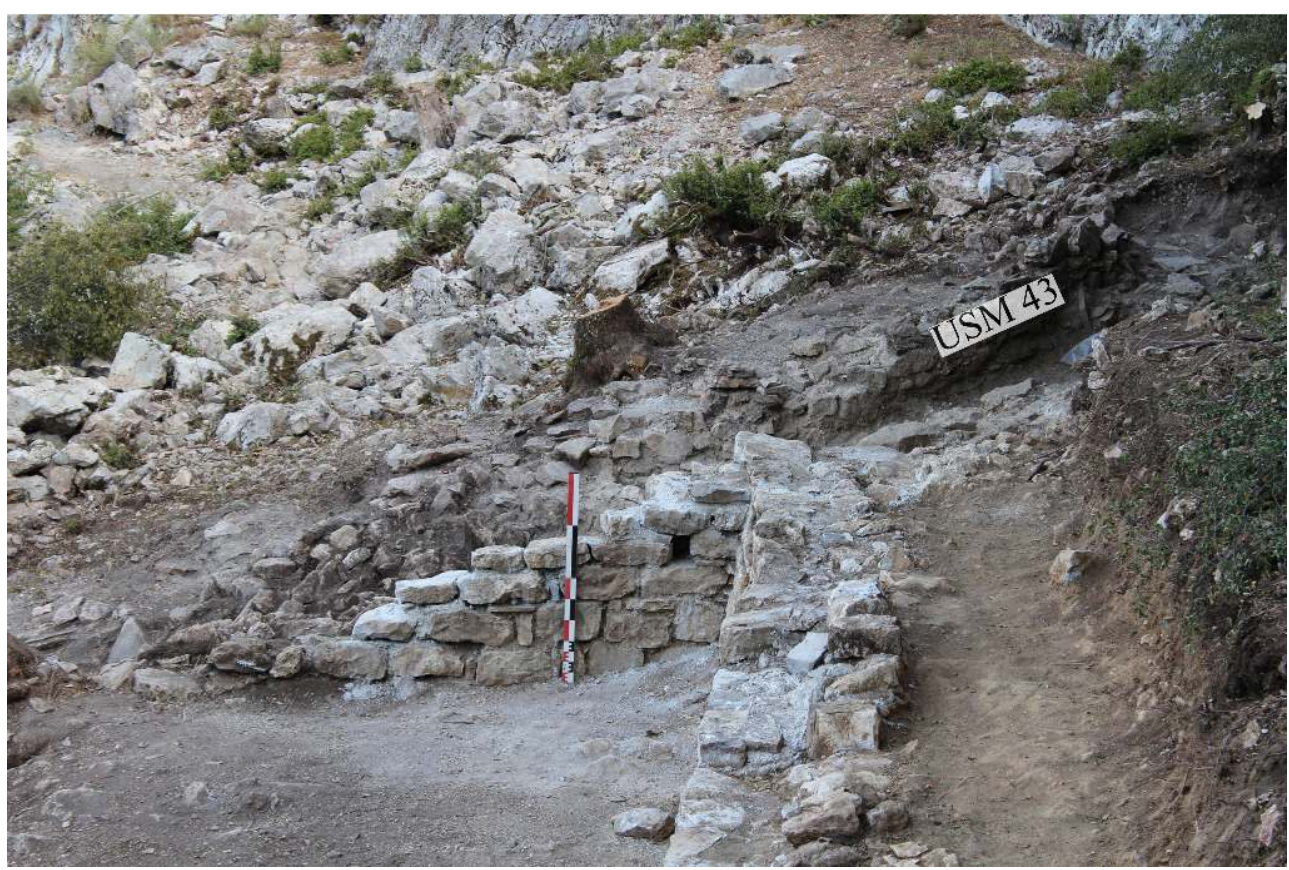

VUe de LA ZONe 1B AVEC Les Vestiges de L'ABside (USM 43), PARTIE NORD.
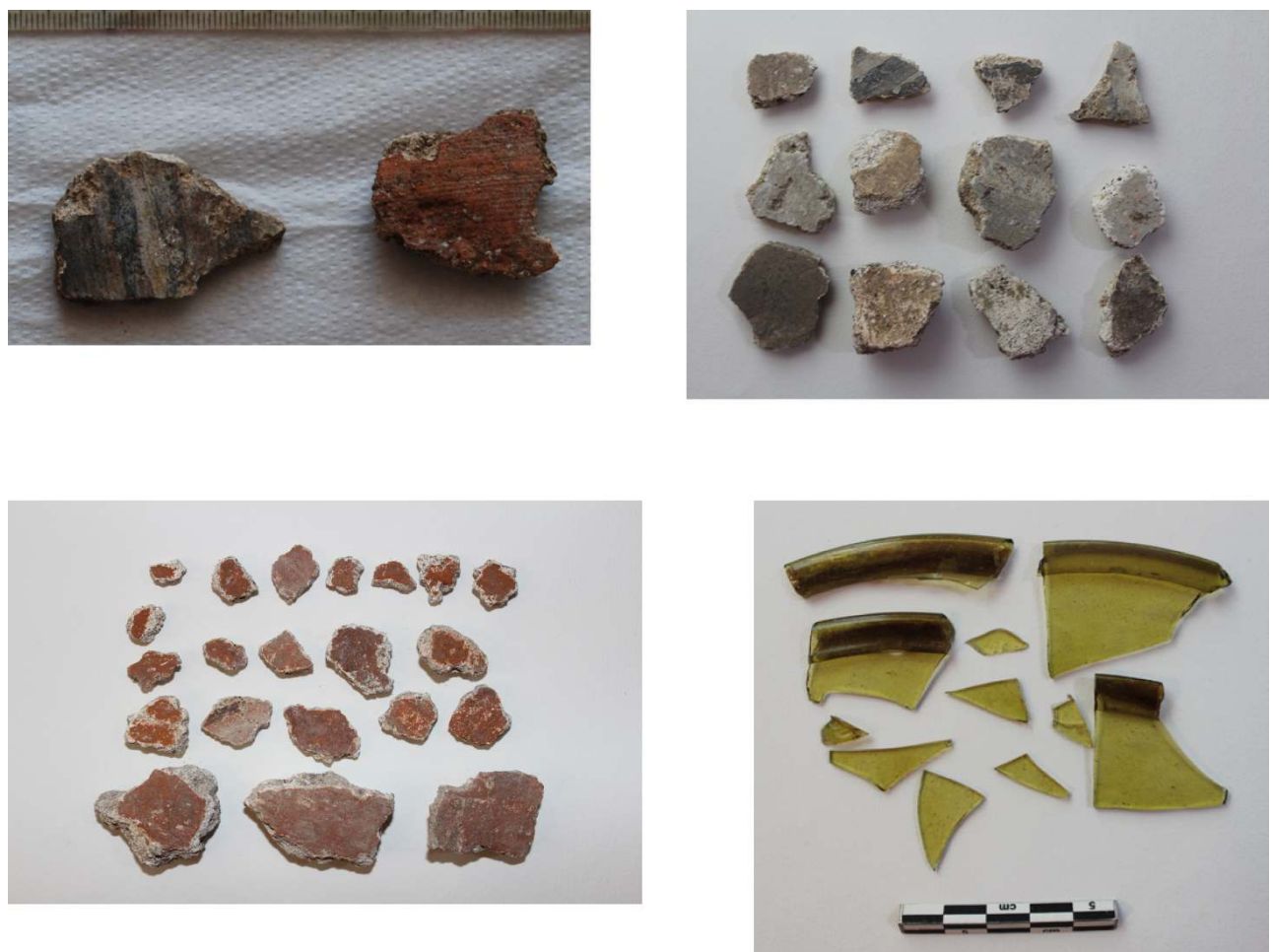

FRAgMentS de PEINTURE MURALE de L'ABSIDE ET d'ASSIETTE EN VERRE.

32 Il comporte trois à quatre assises de pierres liées au mortier de chaux et se dirige vers l'est, suivant la pente du terrain. Il s'agit des vestiges de l'abside d'une église, dont les niveaux supérieurs de l'espace interne ont livré de nombreux petits fragments de peinture murale. À l'extérieur, à une distance de 0,10 à $0,15 \mathrm{~m}$ de l'abside, un mur de pierres liées au mortier paraît avoir été rasé (USM 44). À cet endroit, trois inhumations 
$(325,326,327)$ ont été identifiées. La sépulture 325 , recouverte de dalles, est installée entre les USM 44 et 43 , tandis que les deux autres, dépourvues de couverture, laissent apparaître des fragments d'inhumation. Leur fouille est prévue pour la prochaine campagne de 2012.

Nous sommes ainsi en présence d'un nouveau quartier de la ville basse (zone 1B), de caractère religieux, installé dans l'ensemble d'une cavité rocheuse dirigée vers l'est et organisé, comme le quartier de la zone médiane $1 \mathrm{~A}$ (secteurs 1 et 2), sur des terrasses. Les aménagements attendus (remparts) ont ainsi laissé la place à des installations ecclésiastiques. Une nouvelle église vient d'être identifiée dans ce quartier, flanquée d'un bâtiment quasi-rectangulaire d'une bonne qualité de construction, dont le caractère monastique semble être une hypothèse plausible. Sa fouille complète sera une des priorités de la prochaine campagne. Cette nouvelle découverte porte à quatre le nombre des églises réparties dans les différentes zones composant le site de Komani et à six leur nombre dans son territoire proche.

Quelques fragments de céramique culinaire, identifiés dans la couche couvrant les inhumations entre les USM 43 et 44 , dateraient assez largement entre le VIII et le XII ${ }^{e}$ siècle ; des fragments d'une assiette de verre ont été également été mis au jour pendant le nettoyage de l'USM 38.

\section{L'église Saint Georges (zones 2) (fig. 12)}

Fig. 12 - Komani. Plan de l'église Saint-Georges et des structures environnantes.

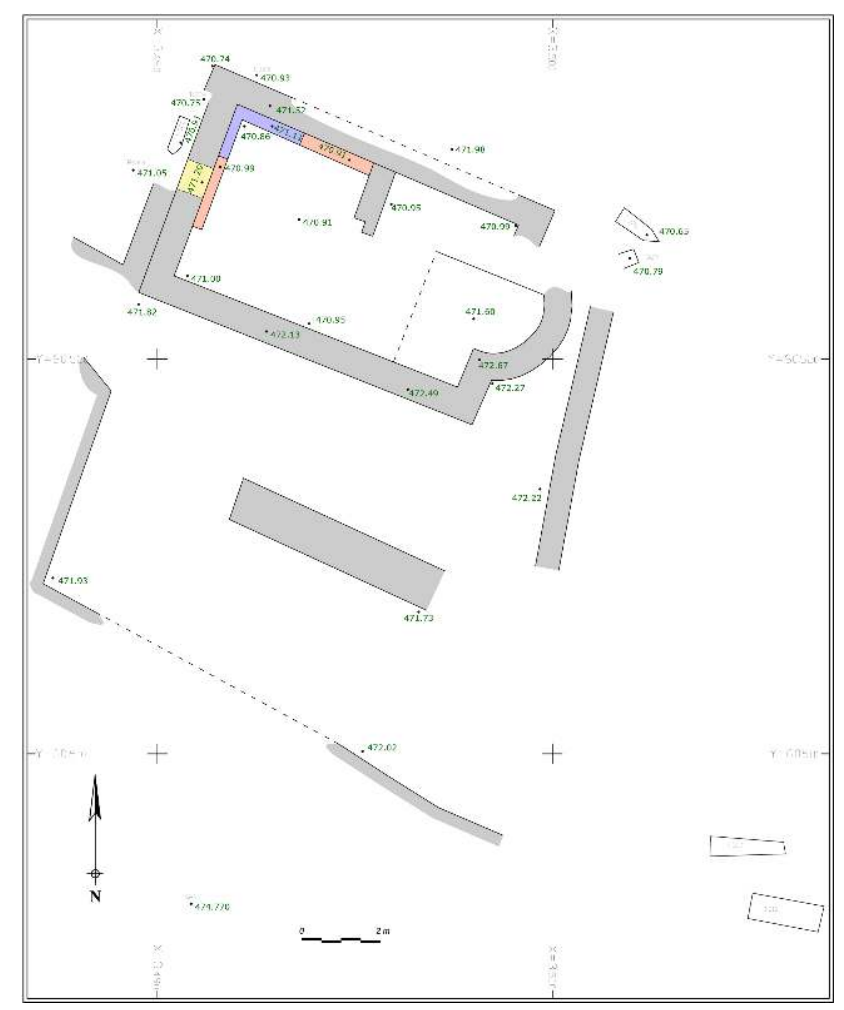

DAO : N. PAIREAU. 
35 À l'issue des résultats de la campagne de 2010, nous avions prévu de consacrer une attention particulière à la zone funéraire 2 et notamment aux abords de l'église SaintGeorges. Pour les raisons évoquées plus haut (note 2), les opérations se sont concentrées sur l'îlot de Saint-Georges et uniquement sur l'étude de l'église même, déjà engagée lors des précédentes campagnes.

Un niveau de sol a été dégagé dans la nef, qui dans son dernier état était pavé de dalles majoritairement en calcaire, prises dans un lit de mortier de chaux conservé par endroits (fig. 13).

Fig. 13 - Komani. Église Saint-Georges, partie occidentale de la nef (dallage, bordures et banc), vue de l'est.

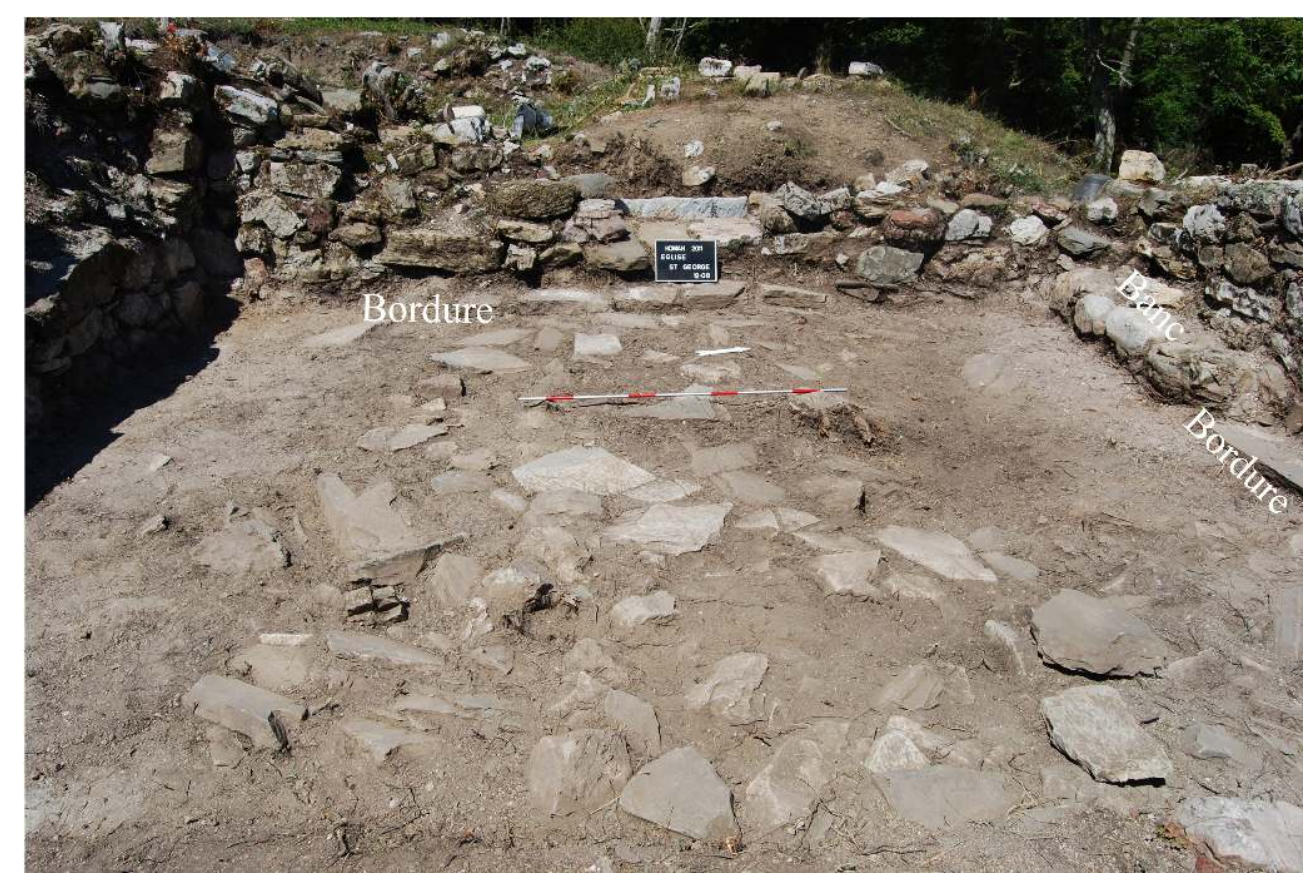

Il est présent dans la partie nord-ouest de la nef, tandis que dans la partie sud-ouest, la roche naturelle a dû rester nue. Une bordure maçonnée de pierres et de dalles (de 0,30 m de large) contourne le bas des murs nord (à partir de l'installation qui limite l'espace sacré) et ouest (à 3,20 m), légèrement plus haute que le niveau de pavement. Les pierres de cette structure semblent être intégrées à la base des murs. Un deuxième banc maçonné de grosses pierres non taillées s'installe sur 0,30 m d'élévation par-dessus cette bordure, sur une longueur de 1,53 m à partir de l'angle nord-ouest, appuyé au mur nord.

L'espace devant l'entrée de la nef a également été fouillé et des travaux importants ont dégagé l'ensemble des matériaux effondrés des élévations au nord de l'église. Plusieurs inhumations se dessinent devant l'entrée (T. 322 et 324), à l'angle nord-ouest du mur nord (T. 323) et près de l'angle nord-est de l'abside (T. 328, 329), dont seulement deux ont été fouillées. La première (T. 322), située au nord de l'entrée, a livré une structure en moellons contenant les ossements de plusieurs individus en position secondaire. La moitié d'une pierre de meule se trouve intégrée dans la structure tombale. La deuxième (T. 323) était juste un dépôt secondaire de très peu d'ossements, et a livré le pendentif en étoile d'une boucle d'oreilles en bronze, datant des VIII ${ }^{\mathrm{e}}-\mathrm{IX}^{\mathrm{e}}$ siècles. 
Un niveau d'usage a été identifié devant l'entrée de la nef mais pas de sol aménagé. Un groupe de sept monnaies byzantines en argent, apparemment du milieu du IXe siècle, collées les unes aux autres, a été découvert dans ce niveau au sud de l'entrée (fig. 14).

Fig. 14 - Komani. Partie nord devant l'entrée de l'église Saint-Georges et mobilier mis au jour.

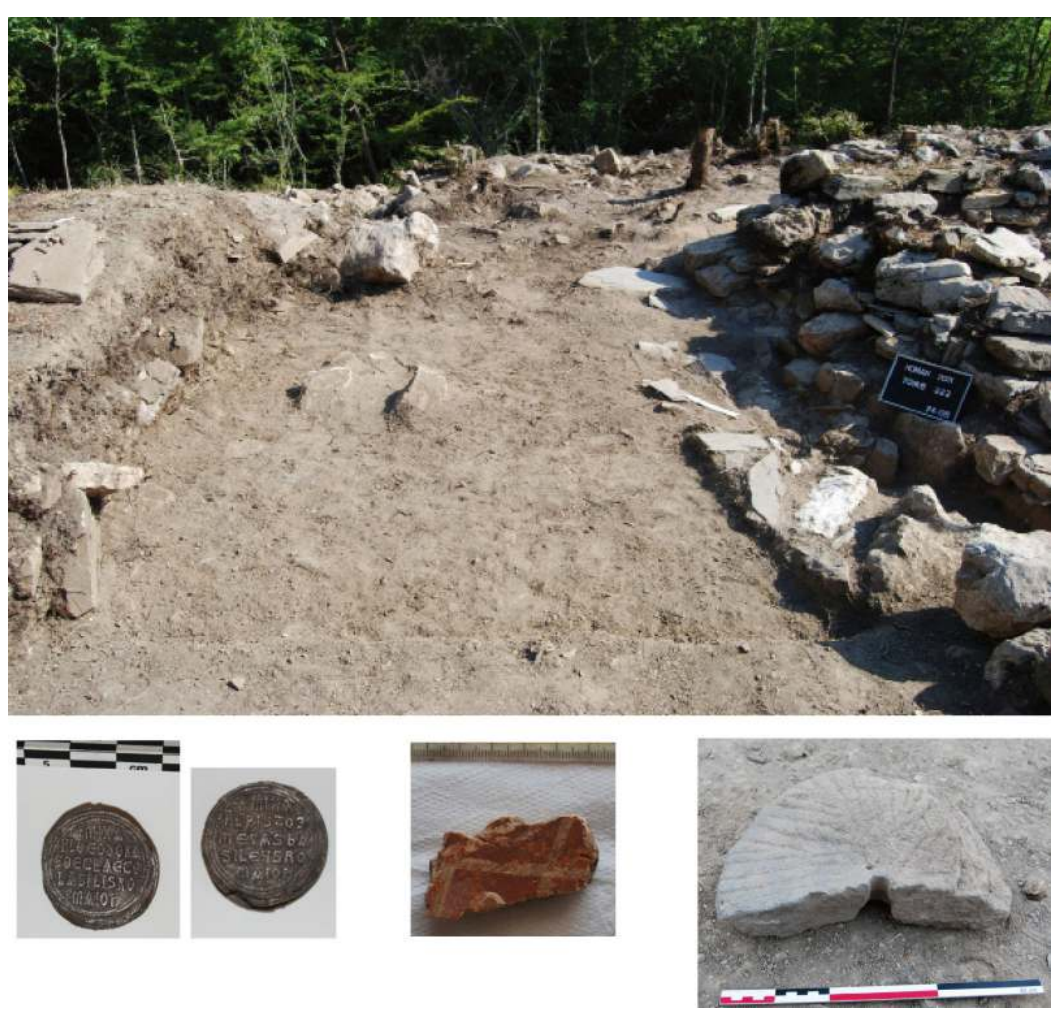

La découverte de nouveaux fragments d'enduits peints renforcent l'hypothèse selon laquelle le bâtiment était recouvert de décors peints dont la densité et la nature restent inconnus en l'état actuel de la recherche et de la conservation des vestiges.

\section{Mise en valeur, préservation du site et conservation du mobilier}

Il convient de noter que Komani constitue un site de montagne, à l'heure actuelle à l'écart des axes, même secondaires, de communication en Albanie. Son importance historique (c'est le site éponyme depuis plus d'un siècle d'une identité culturelle des premiers siècles du Moyen Âge dans l'occident balkanique), appuyée par les résultats de la mission de recherches archéologiques franco-albanaise depuis trois ans, ont conduit les autorités albanaises à engager une action de valorisation du site. L'apport primordial consiste à intégrer le site dans le réseau de communication. Nous avons engagé :

1. La constitution du dossier de mise en valeur du site, concernant le suivi des modalités nécessaires à la construction d'une route communale conduisant au site ${ }^{5}$.

2. L'aménagement du site même et son organisation en vue d'une prochaine ouverture aux visiteurs. L'envahissement de la majorité des zones composant le site par la végétation forestière a accéléré considérablement la destruction des vestiges. L'absence d'entretien a conduit à la quasi-disparition des structures sous la végétation, d'une campagne à l'autre. De ce fait, des travaux importants de déforestation, de nettoyage et de consolidation des 
structures fouillées ou endommagées ont été engagés non seulement pendant la durée de notre mission de terrain, mais aussi toute l'année. L'ensemble des structures bâties mis au jour dans la zone 1B a été consolidé, ainsi que les murs endommagés par la végétation dans le secteur de l'église de Saint-Georges.

L'aménagement des voies de circulation sur le site a privilégié cette année deux sentiers : le principal qui monte du village de Komani vers la zone de l'habitat 1 et un secondaire qui relie l'église Saint-Georges avec le sentier habitat-nécropole. Des travaux importants de déforestation ont été engagés pendant la la durée de la mission, principalement de la zone entre l'habitat $1 \mathrm{~B}$ et la nécropole, en vue de l'aménagement et du stockage des matériaux de construction.

Il est envisagé de faire appel en 2012 à un spécialiste, pour permettre de faire le point sur l'état de la conservation des structures, établir la priorité des travaux de sécurisation, et aider dans l'élaboration d'un projet de mise en valeur des structures bâties dans un environnement densément boisé. Nous envisageons la mise en place d'un réseau de communications entre quartiers, ces derniers équipés d'un système signalétique approprié. Cela impliquerait la collaboration de la mission de Komani avec le Directorat des Monuments historiques de Tirana et de Shkodra en fonction de critères à la fois scientifiques et de mise en valeur.

3. Une importante opération de restauration du mobilier (métaux, céramique et verre), principalement de provenance funéraire, conduite par le restaurateur E. Bitri. Il est prévu de présenter ce mobilier d'une exceptionnelle richesse, conjointement à celui de Lezha, dans une exposition illustrant le haut Moyen Âge balkanique à travers sa spécificité nordalbanaise en 2012.

\section{BIBLIOGRAPHIE}

Garam 1987 = E. Garam, Der awarische Fundstoff im Karpatenbecken und seine Gliederung, dans

B. Hänsel (dir.), Die Völker Südosteuropas im 6. bis 8. Jahrhundert, Berlin, 1987 (Südosteuropa-Jahrbuch, 17), p. 191-201.

Pletneva 1989 = S. A. Pletneva, Na slaviano-hazarskom pogranitche [La frontière entre les Slaves et les Khazars], Moscou, 1989, p. 96.

\section{NOTES}

1. Nos remerciements pour ce programme vont spécialement à : S. Gioanni, directeur des Études médiévales de l'École française de Rome; R. Chaffort, Attaché culturel de l'Ambassade de France en Albanie ; Sh. Gjongecaj, directrice de l'Institut archéologique d'Albanie ; A. Marashi, directeur du Centre des études albanologiques, pour leur soutien et leur disponibilité. Nous remercions également la direction de l'Albanian Development Fund pour son implication particulière dans la construction de la voie communale conduisant au site.

2. L'intervention d'un spécialiste de la sécurité de chantiers en montagne est prévue pour la prochaine campagne de terrain, afin de pouvoir assurer l'exploitation de la zone 1 (habitat), en particulier la partie sommitale de la citadelle; une partie de notre équipe, en charge du dossier 
funéraire, a été mobilisée sur le chantier de Lezha, codirigé par Luc Buchet et Etleva Nallbani. Les travaux de la construction de la route menant à la citadelle de Lezha (entrepris par l'état albanais) ont nécessité des fouilles de sauvetage, assurées par cette partie de notre équipe en partenariat avec l'AshA.

3. Garam 1987, p. 191-201 ; Pletneva 1989, 1989, p. 96.

4. MEFRM 122-2, 2010, p. 471-484 (chronique Komani).

5. Un rapport sur les valeurs archéologique du site de Komani et son impact d'une part sur la valorisation du patrimoine médiéval balkanique et d'autre part sur le développement économique et social des communautés locales, a été déposé auprès du gouvernement albanais. Cela a donné suite à l'intervention de l'Albanian Development Found, qui a prévu d'initier les travaux au printemps de l'année 2012. Les opérations de diagnostic archéologique, en partie engagées en 2011, sont à mener en collaboration avec l'AshA (du Ministère du tourisme et de la culture albanais) destinée à piloter les travaux.

\section{INDEX}

Index géographique : Albanie, Komani

Mots-clés : Adriatique, haut Moyen Âge, nécropole, peuplement, anthropologie, échanges institutions Ministère des Affaires étrangères et européennes (Paris), École française de Rome, Institut archéologique d'Albanie (Centre d'études albanologiques - Tirana), INED (Paris), Centre d'histoire et civilisation de Byzance (UMR 8167 - « Orient et Méditerranée » Paris)

\section{AUTEURS}

ETLEVA NALLBANI

C.N.R.S., Orient et Méditerranée - UMR 8167 - etlevanallbani[at]gmail.com

$$
\text { MAËL JULIEN }
$$

DAPCAD

\section{ELVANA METALLA}

Institut archéologique de Tirana - emetalla[at]hotmail.com 